\title{
DESIGN DE DISPOSITIVOS MÉDICOS: CONTRIBUTO DO DESIGN PARA O DESENVOLVIMENTO DE UMA PRÓTESE EXTERNA DE UM MEMBRO INFERIOR.
}

\section{DESIGN OF MEDICAL DEVICES: DESIGN CONTRIBUTION FOR THE DEVELOPMENT OF A PROSTHESIS OUTSIDE OF A LOWER LIMB.}

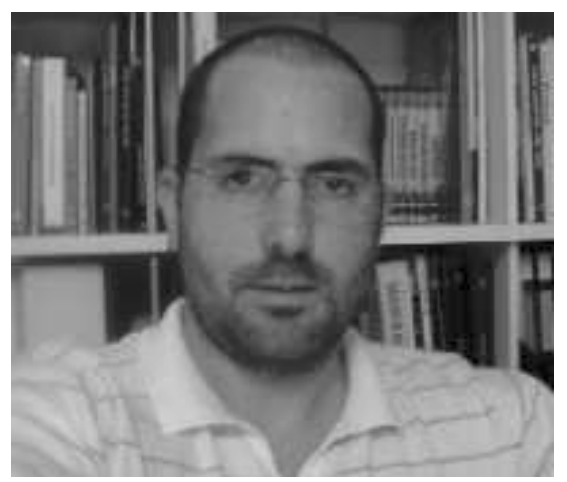

\section{Demétrio Ferreira Matos}

Mestre em Design Industrial pela Faculdade de Engenharia da Universidade do Porto

Professor convidado. Departamento de Design do Instituto Politécnico do Cávado e do Ave. Portugal

dmatos@ipca.pt

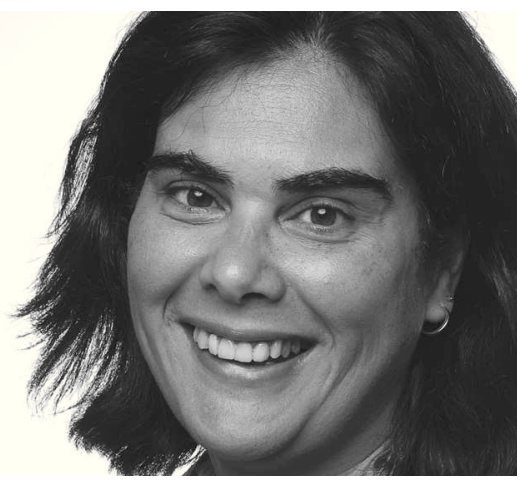

\section{Ana Margarida Ferreira}

Doutorada em Engenharia da Produção pela Universidade da Beira Interior.

Professora Auxiliar no IADE-U, Portugal. Coordenadora da Licenciatura em Design e Coordenadora do Grupo de Investigação ID:Co.Lab da UNIDCOM/IADE.

ana.margarida.ferreira@iade.pt 

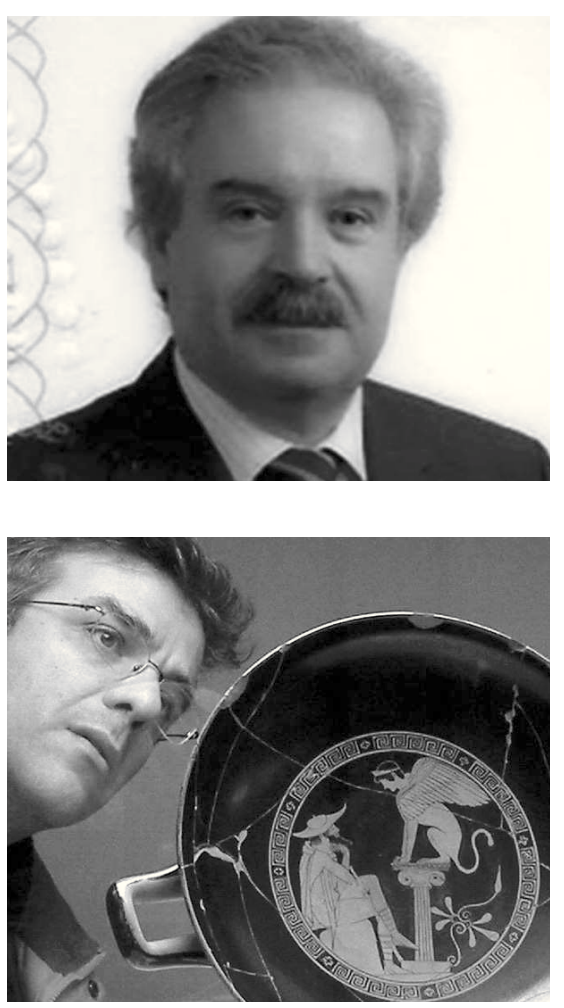

\section{António Marques Pinho}

Doutorado em Engenharia Mecânica.

Professor Auxiliar da Escola de Engenharia da Universidade do Minho, Portugal. Departamento de Engenharia Mecânica. Membro do MEtRICs Research Center acmpinho@dem.uminho.pt

\section{João Paulo Martins}

Doutorado em Arquitectura pela Universidade Técnica de Lisboa.

Professor Auxiliar da Faculdade de Arquitectura da Universidade Lisboa, Portugal. Departamento: Projecto de Arquitectura, Urbanismo e Design. Membro do CIAUD

martins@fa.ulisboa.pt

\section{RESUMO}

Conscientes da importância das abordagens sistémicas, multidisciplinares e centradas no usuário para o desenvolvimento e sucesso de novos produtos no âmbito da saúde, nomeadamente de próteses endosqueléticas para membros inferiores, desenvolveu-se uma investigação ativa recolhendo e validando, através de métodos como a crítica literária, a observação direta, a aplicação de questionários, a realização de entrevistas e vídeos ou, ainda, a concepção e teste de protótipos, as variáveis necessárias a uma resposta mais eficaz e global do novo produto. Os resultados desta investigação permitem, num primeiro plano, ultrapassar limitações destes dispositivos na reprodução do ciclo de marcha e obter soluções mais satisfatórias na fase inicial da reabilitação e durante a vida activa do paciente. Como última e principal consequência, permitem a melhoria da qualidade de vida dos amputados.

\section{PALAVRAS-CHAVE}

Design para a Saúde. Prótese externa do membro inferior. Qualidade de vida. 


\section{ABSTRACT}

Aware of the importance of systemic approaches, multidisciplinary and centered to user for the development and success of new products in the health, including endoskeletal prosthesis for lower limbs, an active research developed by collecting and validating, through methods such as literary criticism, direct observation, questionnaires, conducting interviews and videos, or even the design and testing of prototypes, the variables necessary for more effective global response and the new product. The results of this research allow, in a first plane, overcome limitations of these devices in the reproduction of the gait cycle and more satisfactory solutions in the initial phase of rehabilitation and during the active life of the patient. As a final and major consequence, allow improving the quality of life of the amputee.

\section{KEYWORDS}

Design for Health. External prosthetic of the lower limb. Quality of life.

\section{INTRODUÇÃO}

O Design para a saúde é uma área com dimensão e importância social global e que se caracteriza hoje por níveis cada vez mais elevados de exigência e competitividade. Perante essa realidade, o objeto deste estudo, que resulta de uma investigação de doutoramento em curso, consiste na importância do processo de design, em particular da abordagem centrada no usuário, e de métodos de investigação a si associados para um incremento do desenvolvimento e dispositivos médicos para a melhoria da qualidade de vida.

Na última década assistiu-se a um maior interesse pelo design de dispositivos médicos, especificamente em relação às questões ligadas à segurança do paciente, e uma série de iniciativas foram criadas com o objetivo de melhorar esses aspectos (MARTIN et al., 2008). Em consequência, emergiu a perceção do desenvolvimento desse tipo de dispositivo ter em conta uma série de variáveis, tais como: o contexto em que são utilizados e o papel que desempenham; incluindo, entre outros, preocupações que se prendem às necessidades dos profissionais de saúde e dos pacientes. Por outro lado, ainda é possível verificar o caso de muitas equipes multidisciplinares responsáveis pelo desenvolvimento destes dispositivos não integrarem designers como profissionais-chave para o sucesso destes projetos. Este argumento é 
reforçado pela perceção de que, com muita frequência, estes produtos não respondem plenamente às necessidades reais de seus usuários. Questões como as funções estético-formais ou simbólicos ou, em casos extremos, questões ergonómicas e funcionais, por exemplo, não são consideradas tão importantes como o desempenho mecânico ou as variáveis econômicas e tecnológicas.

Em todo o mundo existem milhões de pessoas com membros inferiores amputados, enfrentando fortes níveis de vulnerabilidade física e psicológica (McGIMPSEY \& BRADFORD, 2010). Neste contexto, com aplicação específica neste trabalho, foi realizado o estudo do universo de pessoas privadas de um membro, seja por amputação traumática ou má formação congénita (BOCCOLINI, 2000; CARVALHO, 2003; LIANZA, 1995), fazendo a substituição do membro através da utilização de um dispositivo protésico; mais precisamente uma prótese endosquelética do membro inferior. Entende-se por prótese uma peça ou aparelho que tem como função substituir parcialmente ou totalmente um membro ou órgão ausente (LIANZA, 1995). Dentro de cada categoria de "deficiência" existem interações entre pessoas e soluções tecnológicas diferentes com objetivo de resolver os problemas específicos inerentes a cada processo de reabilitação. Neste contexto, o papel do design não deve ser apenas perspectivado como um instrumento de diferenciação do produto, dando-Ihe competitividade pela ligação à moda, por exemplo; mas como uma proposta de design inclusivo e sustentável, no qual existe o compromisso com uma resposta holística do produto e visando, antes de tudo, o bem-estar e a qualidade de vida (FERREIRA, 2003) da pessoa amputada no maior período ou ciclo de vida do produto possível.

De modo a enquadrar e perceber as relações entre todos estes conceitos, desenvolveu-se o esquema apresentado na Figura 1, no qual é possível perceber questões como o design e os aspectos que the são inerentes, sua competência e contributo para as equipes multidisciplinares de desenvolvimento de produto. $O$ esquema também apresenta a definição de dispositivos médicos, as normas a si respeitantes e sua importância no desenvolvimento deste tipo de processo, questões ligadas à amputação e à reabilitação protética e a todo o seu universo. 
Figura 1: Universo de desenvolvimento da prótese

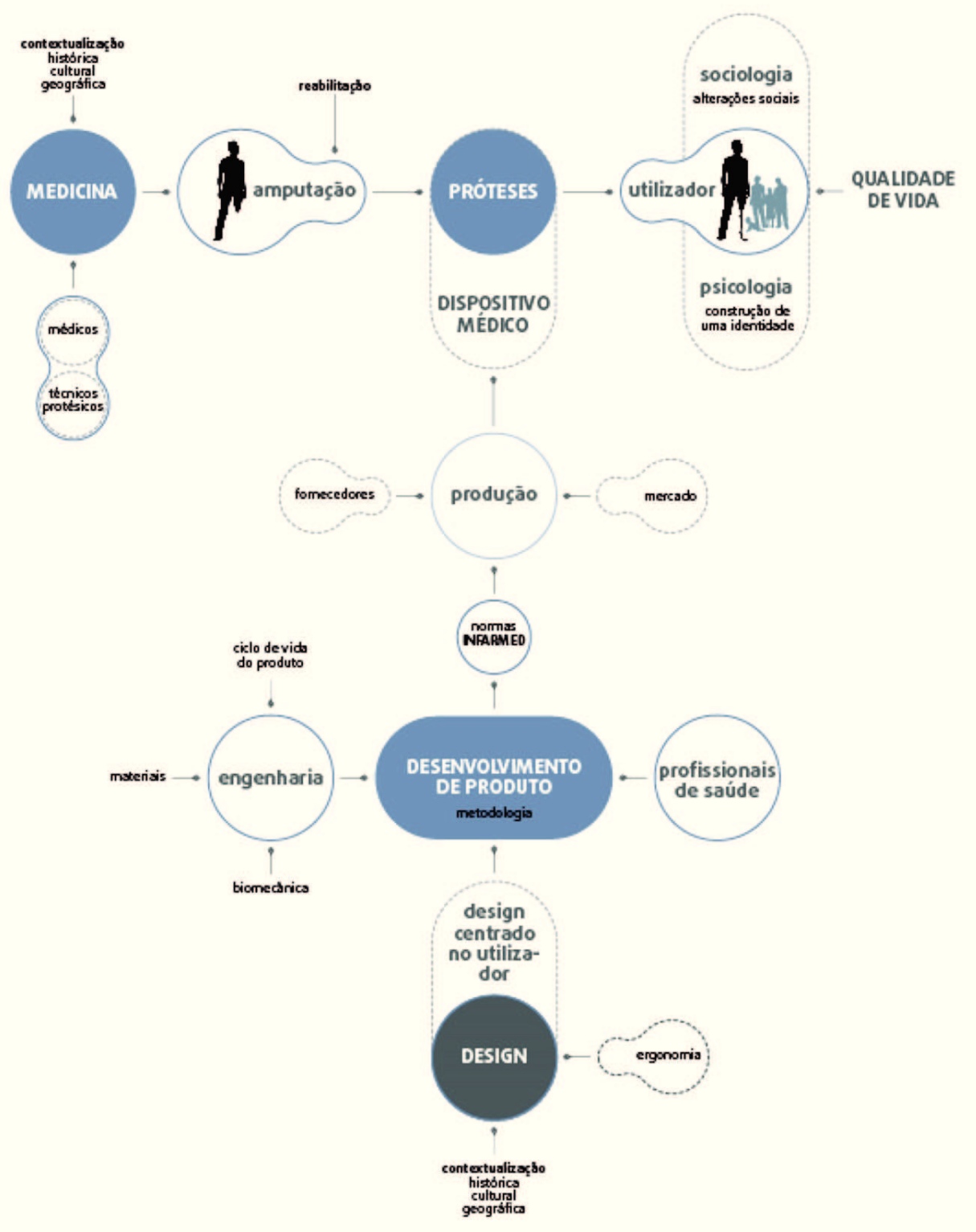

Fonte: produzido pelo autor.

Para a melhor sequência do processo de investigação, verificaramse três grandes momentos metodológicos, a saber, uma primeira fase de análise e perceção do problema em causa, uma segunda fase de síntese e, finalmente, uma terceira de validação. No primeiro momento metodológico uma metodologia qualitativa de base não-intervencionista foi utilizada, incluindo a observação direta dos usuários e processos envolvidos; 
apoiada, entre outros, pela revisão de literatura, com o objectivo de definir o problema de forma mais eficaz. Para viabilizar todo este processo nos seus vários momentos foram estabelecidas parcerias com o Centro de Reabilitação Profissional de Gaia [1] e com a Associação dos Deficientes das Forças Armadas [2], fundamentais para o acesso às fontes primárias de informação, nomeadamente os usuários finais destes produtos e os técnicos ortoprotésicos. Estas parcerias permitiram um estudo in loco e em profundidade das interações diárias entre produto/usuários, registado por diferentes meios.

\section{ANÁLISE}

Os dispositivos médicos constituem um grupo diversificado de produtos que vão desde artefatos simples como luvas e seringas, a dispositivos complexos como um coração artificial (FERREIRA, 2008). Atualmente, as próteses endosqueléticas são compostas por vários componentes que são adquiridos e montados tendo em conta as necessidades e a tipologia do usuário (Figura 2). Neste caso, destaca-se o fato dos componentes não são usados de forma independente mas como parte de um sistema.

Figura 2: Componentes da prótese.

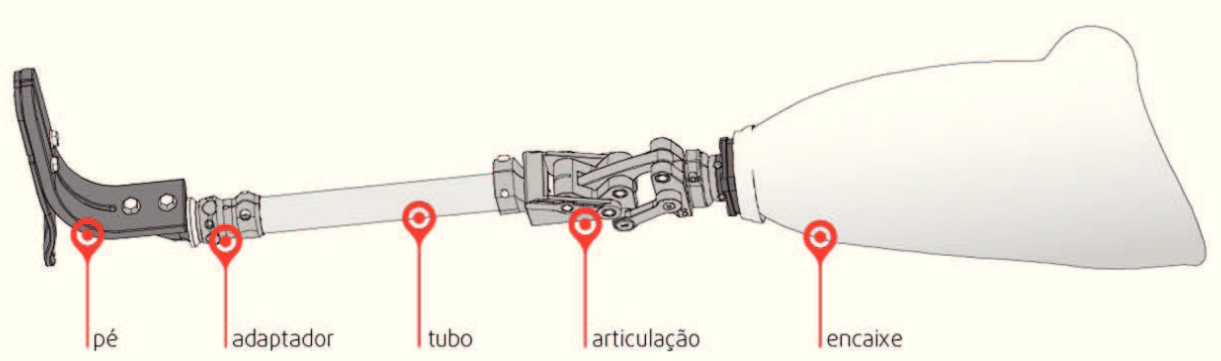

Fonte: MATOS, 2009

Apenas os encaixes das próteses são realizados nos centros ortopédicos. Esta situação explica-se pelo fato destes produtos serem personalizados; ou seja, cada amputado tem um coto com as suas características geométricas, o que requer cuidados específicos. É também importante referir que existe 
uma grande preocupação por parte de alguns amputados para que o aspeto estético da sua prótese se aproxime do aspeto do membro residual. Para responder a esta questão, existem acabamentos cosméticos que permitem revestir toda a estrutura de uma prótese endosquelética. Estes acabamentos são feitos, na maior parte dos casos, por uma espuma de poliuretano (Figura 3) ou por um revestimento em silicone (Figura 4) que simulam o volume do membro e que visam imitar o membro saudável. Em ambos os casos, há um aumento do custo final do dispositivo, particularmente na última solução (revestimento em silicone).

Figura 3: Revestimento cosmético em poliuretano.
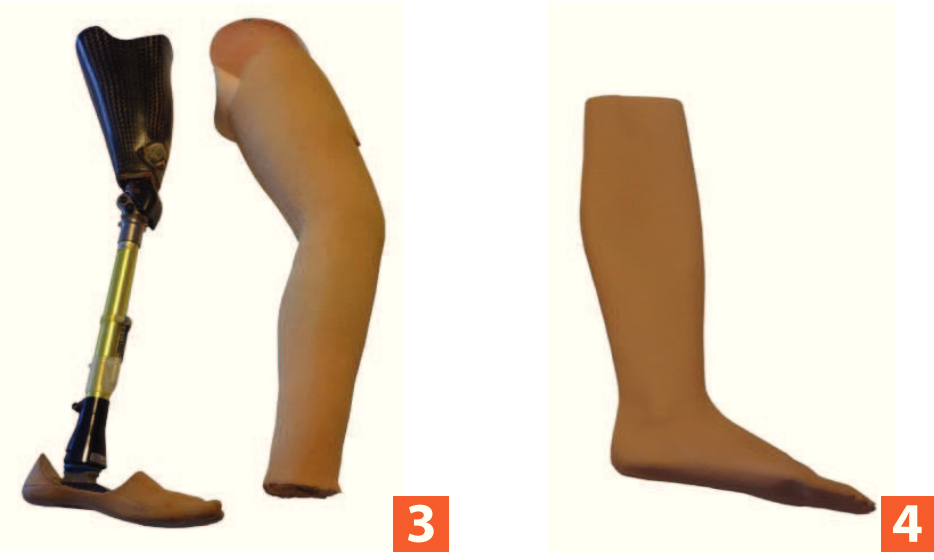

Figura 4: Revestimento cosmético em silicone.

Fonte: Imagens do autor.

Após a realização de entrevistas semi-estruturadas com os potenciais usuários, foi desenvolvido um questionário, funcionando como pré-teste, com o objetivo de identificar e analisar situações mais relevantes para esta investigação. Sendo a amostra dos amputados e sua proveniência fatores cruciais (CRESWELL, 2009; HILL \& HILL, 2009; PEREIRA, 2002; QUIVY \& CAMPENHOUDT, 1998), foi dedicado um longo período de tempo para ultrapassar a dificuldade de acesso aos pacientes, bem como para afinar a complexa caracterização dos usuários; sublinhando, entre outras questões, a falta de dados sobre o número de amputados em Portugal. Na realidade, pode afirmar-se que existem poucos dados a nível nacional sobre o número de amputações uma vez que são habitualmente incluídas sob a designação de "deficiências motoras" (OLIVEIRA, 2004). Repara-se que nos dois únicos estudos publicados em Portugal verificou-se que a maior parte 
das amputações são de origem vascular, causas traumáticas, seguidas da insuficiência arterial crônica. Para superar essas limitações, foi realizado um pré-teste com pacientes do Centro de Reabilitação Profissional de Gaia, no qual foram abordadas questões abertas e fechadas a fim de obter respostas de natureza qualitativa e quantitativa. Depois de analisar o questionário, a versão final do mesmo, com a redução de questões abertas, foi aplicada aos membros da Associação dos Deficientes das Forças Armadas. A interpretação destas respostas permitiu otimizar as situações abordadas através de perguntas fechadas. $\mathrm{O}$ exercício metodológico permitiu reduzir o número total de problemas e otimizar a leitura do questionário.

Os resultados do questionário aplicado foram conclusivos mostrando que o desenvolvimento de uma solução de inovação incremental será mais bem-sucedido no contexto português do que o desenvolvimento de uma solução de inovação radical. Também foram incluídas perguntas sobre conforto, funcionalidade, estética e preço dentro de uma sequência lógica de preocupações dos pacientes. Estas preocupações surgiram casualemte, associadas ao estado dos pacientes e à sua fase de reabilitação. Em termos gerais é possível afirmar que, na fase inicial, as pessoas estão mais preocupadas com a recuperação da função perdida, neste caso a marcha. Depois do domínio, já com alguma destreza, das suas próteses na realização de tarefas cotidianas, numa segunda fase, os usuários destes equipamentos médicos assumem preocupações mais centradas na dimensão estética.

\section{SÍNTESE}

De acordo com Ulrich e Eppinger (2008), o desenvolvimento de produtos corresponde a uma sequência de atividades que uma empresa deve seguir para criar, produzir e vender um produto. É igualmente uma atividade interdisciplinar que requer a participação de quase todos os membros de uma organização ou equipe. Para os autores, na fase de síntese, é fundamental uma particular atenção na aplicação da metodologia de design centrada no usuário, uma vez que o seu resultado será posteriormente validado pelo destinatário do produto, processo ou sistema alvo de projeto. Este método deve ser visto como uma abordagem que articula o envolvimento do designer e do usuário (IDEO, 2009) na fase de projeto. No caso do processo de investigação em curso, foram consideradas as variáveis resultantes desse envolvimento, assim como variáveis relativas às matérias normativas. São 
de referir as apresentadas pelo Infarmed, pelo ISO 10328: 2006 (Próteses - O teste estrutural de próteses de membros inferiores - Requisitos e métodos de ensaio) e pela Lei Portuguesa no 46/2006, de 28 de Agosto, sobre as prescrições regulamentares essenciais e sobre o desenvolvimento e avaliação clínica destes dispositivos (INFARMED, 2010; International Organization for Standardization. ISO 10328, 2006). A quantidade e complexidade das variáveis identificadas neste projeto reforçaram a perceção inicial da necessidade de uma equipe multidisciplinar para a melhor sequência e resposta da solicitação de projeto.

Figura 5: Fase de desenvolvimento conceitual.
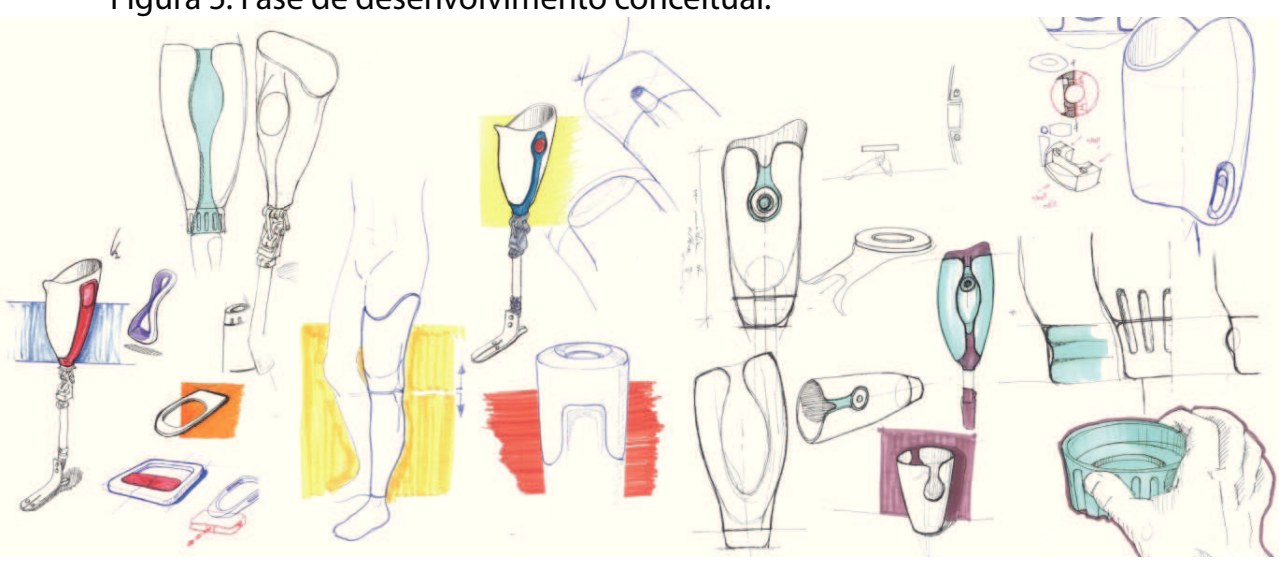

Fonte: dos autores.

Merece ainda ser mencionado que, em termos de processo de criação e desenvolvimento da nova solução de prótese, o projeto desenrolou-se em duas fases. A primeira fase tratou com particular enfoque a resposta à função prática. Nela, questões de uso, de desempenho funcional e mecânico, entre outros, foram tratados com especial atenção. Na segunda fase, foram consideradas as questões relativas à função estético-formal e à função simbólica (BÜRDEK, 2006). Estas últimas estão diretamente relacionadas ao "tratamento da pele" do produto em causa. Idealmente, a prótese deve ser funcional de modo a ajudar o amputado na sua atividade física. Deve ser confortável, fácil de colocar e remover, leve, agradável esteticamente e ter preços acessíveis. Para além destes fatores, as próteses devem ser sustentáveis, cumprindo, entre outros, um ciclo de vida prolongado.

A resposta a estas preocupações fez emergir com particular destaque o exercício da modularidade do dispositivo protésico do membro inferior.Como 
exemplo, esta solução permite ao usuário a alteração do dispositivo médico de forma rápida e fácil (Figura 6) através de um encaixe. Um dos objetivos do projeto passa a ser, então, a capacidade de articular e proporcionar uma desconexão rápida entre o encaixe e os outros componentes protéticos que compõem o sistema sob estudo.

Figura 6: Fase 01. Conceito de modularidade.

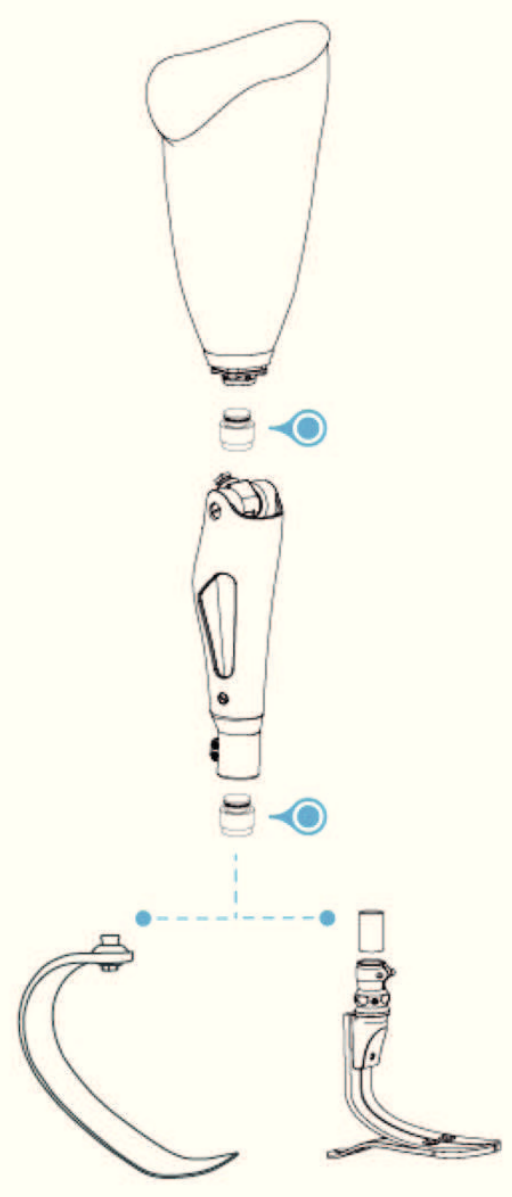

Fonte: dos autores.

Tal como já foi referido, uma série de requisitos a cumprir pelo produto foram atendidos. Entre eles, a segurança e qualidade do desempenho do dispositivo protésico, consideradas em diferentes ambientes e em situações extremas de impacto. Este cuidado resulta da importância do sistema permitir ao usuário a realização de qualquer atividade física, se possível sem condicionantes. Assim, a robustez, a resiliência do sistema em vários ciclos 
de utilização e fiabilidade do mesmo são evocados, de modo a assegurar o melhor e mais duradouro funcionamento. O alinhamento da prótese, definido pelos técnicos, a densidade do aparelho, limitador da mobilidade do usuário e o custo final prótese são fatores-chave na compra da maioria dos produtos e parâmetros e também não podem ser desconsiderados no processo de criação.

Uma vez melhor definidos os objetivos da investigação e os requisitos do produto, foram desenvolvidas algumas hipóteses de solução em concordância com o que se tinha desenvolvido previamente. Destas hipóteses, algumas foram modeladas e a mais promissora foi prototipada de modo a validar o seu desempenho e comportamento em condições de carga. Realizaram-se testes experimentais com base nos requisitos regulamentados na norma internacional ISO 10328 (2006) que oriente os requisitos para o métodos de ensaio e o teste estrutural de próteses de membros inferiores. Foram também desenvolvidos ensaios estáticos de prova e análises cíclicas de resistência à compressão e à flexão e ensaios ao limite de flexão e torção (Figura 7).

Após a realização destes ensaios e numa perspectiva de melhoria do desempenho do produto, o protótipo foi redimensionamento de acordo com os resultados experimentais e as previsões analíticas feitas.

Figura 7: Ensaio cíclico de flexão.

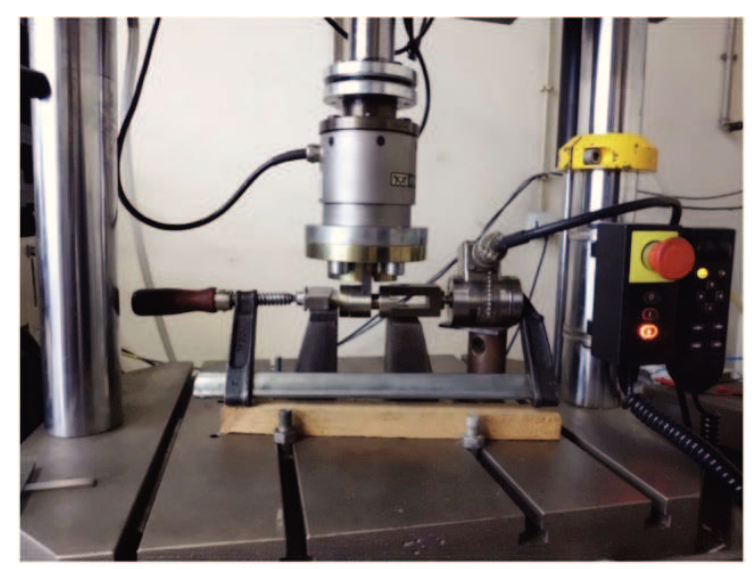

Fonte: imagem do autor.

Com o auxílio do software da Granta Design, o CES Edupack (v.2005), e tendo em atenção a ponderação das características e propriedades 
do dispositivo protésico numa perspectiva do seu bom desempenho e atratividade foi promovida a seleção de materiais. Neste caso, obteve destaque para o projeto o titânio (Ti) comercialmente puro (Ticp) e as suas ligas. Neste contexto, evidencia-se o Ticp, de grau 4, que associa resistência mecânica e baixo custo, comparativamente às ligas de Ti. Esta solução permite igualmente alcançar uma redução de massa.

\section{AVALIAÇÃO}

Um dos principais objetivos da ergonomia consiste em adaptar o produto ao usuário (PHEASANT, 2003), através da compreensão clara da interação entre objeto e usuário. Esta compreensão visa garantir o conforto, bem-estar, eficiência e segurança do novo produto. Este conhecimento tornase particularmente importante na fase de validação do produto, o que deve acontecer pelos seus reais usuárioes, e enquadra-se no que habitualmente é designado por estudo da usabilidade do produto. De acordo com Tullis e Albert (2008), existem várias definições para o termo usabilidade. No entanto, três variáveis recorrentes são sempre identificadas: (1) o usuário envolvido, (2) a realização de uma atividade e (3) a utilização de um produto ou sistema. Esta consciência reforça a necessidade de centrar o processo no usuário. Por essa razão e para dar a melhor sequência ao processo de investigação em curso, a equipe voltará a realizar trabalho de campo e testar os protótipos desenvolvidos junto a um conjunto de amputados.

\section{CONCLUSÕES}

Geralmente as questões de uso representam apenas uma dimensão do produto (KASPER, 2009). No entanto, na conceção de uma prótese, que é entendida como uma extensão do corpo, torna-se importante que se assuma uma abordagem holística. A qual deve ser incorporadas as funções estéticas, simbólicas e de sustentabilidade a fim de tornar a resposta final mais efetiva. De fato, a importância de considerar as necessidades e expetativas do usuário também tem sido reconhecida como tendo uma série de benefícios relacionados com a saúde (MARTIN, 2010). Assim, o processo descrito neste artigo, embora ainda fase de conclusão, dá ênfase ao usuário, à abordagem de design focada no usuário e à premência das questões afloradas para produtos mais eficazes no domínio da saúde; em particular, das próteses estudadas. 
Reconhecendo a importância de promover uma concepção adequada a cada contexto, mas global na sua abrangência, de modo a garantir um dispositivo médico bem-sucedido e o fato da qualidade, segurança e eficácia do dispositivo serem estabelecidas durante a fase de projeto (COLVIN, 2010), espera-se demonstrar com a conclusão desta pesquisa que a contribuição do designer é fundamental para agregar valor ao produto e contribuir para o conforto, bem-estar e qualidade de vida dos usuários de próteses de membro inferior.

\section{NOTAS}

[1] O Centro de Reabilitação Profissional de Gaia (CRPG) é especializado em consultoria, serviços de apoio à integração profissional, tecnologias e serviços de reabilitação. Tem como principal objectivo apoiar a (re) integração na vida activa e profissional das pessoas que recorrem aos seus serviços. www.crpg.pt

[2] A Associação dos Deficientes das Forças Armadas (ADFA) www.adfaportugal.com

\section{BIBLIOGRAFIA}

BOCCOLINI, F. Reabilitação - Amputados, Amputações e Próteses. 2a ed. São Paulo: Robe, 2000.

BÜRDEK, B. E. Design: história e teoria e prática do design de produtos. São Paulo: Edgard Blucher, 2006.

CARVALHO, J. Amputações de membros inferiores: em busca da plena reabilitação. 2a ed. São Paulo: Manole, 2003.

COLVIN, M. An Effective Design Process for the Successful Development of Medical Devices. Design, 2010.

CRESWELL, J. W. Research Design. Qualitative, quantitative, and mixed methods approaches. 3a ed. Califórnia: Sage Publications, 2009.

FERREIRA, A. M. Design e Inovação: Valores para o século XXI, Revista Idade da Imagem, Lisboa, 8, p. 52-56, 2003.

FERREIRA, A. M. Caracterização e Quantificação da Inovação no Processo Evolucionista do Design: análise de um século da prática médico-cirúrgica em Portugal. Dissertação para a obtenção do grau de Doutor em Engenharia de Produção. UBI: Covilhã, 2008.

HILL, M.; HILL, A. Investigação por questionário. 2a ed. Lisboa: Edições Sílabo, 
2009.

IDEO. Human-Centred Design Toolkit: A Free Innovation Guide for Social Enterprises and NGOS Worldwide. San Francisco: IDEO, 2009.

INFARMED. Autoridade Nacional do Medicamento e Produtos de Saúde, I. P. 2010. Disponível em: <http://www.infarmed.pt>. Acesso em 20/10/2010.

Bruxelas, CEN. ISO 10328. Prosthetics. Structural testing of lower-limb prostheses. Requirements and test methods. Especifica os procedimentos para testes de resistência estática e cíclicos sobre próteses de membros inferiores. International Organization for Standardization, Bruxelas, Out. 2006, p.136.

KASPER, C. P. Além da função, o uso. Revista Arcos Design, Rio de Janeiro, n.5, p. 18-24, 2009.

LIANZA, S. Medicina da reabilitação. Rio de Janeiro: Guanabara Koogan, 1995.

MARTIN, J. L. Design for patient safety: User testing in the development of medical devices. London: National Patient Safety Agency, 2010.

MARTIN, J. L. et.al. Medical device development: the challenge for ergonomics. Applied Ergonomics Journal, n.39 (3), p. 271-283, 2008.

MATOS, Demétrio. Dispositivos Protésicos Exteriores: Estudo, Desenvolvimento, Produção, Ensaio e Certificação. 2009. Dissertação (Mestrado em Design Industrial) - Faculdade de Engenharia da Universidade do Porto \& Escola Superior de Artes e Design de Matosinhos, Porto.

MCGIMPSEY, G.; BRADFORD, T. Limb Prosthetics Services and Devices - Critical Unmet Need: Market Analysis. Worcester: Bioengineering Institute Center for Neuroprosthetics, 2010.

OLIVEIRA, R. O sujeito e o corpo perante a incapacidade física. Revista Portuguesa de Psicossomática, Portugal, n.6 (1), p.63-67, 2004.

PEREIRA, A. Guia prático de utilização do SPSS - Análise de dados para ciências sociais e psicologia .3a ed. Lisboa: Edição Sílabo, 2002.

PHEASANT, S. Bodyspace. Anthropometry, Ergonomics and the Design of Work. $2^{\text {a }}$ ed. London: Taylor \& Francis, 2003.

QUIVY, R.; CAMPENHOUDT, L. Manual de investigação em Ciências Sociais. $2^{2}$ ed. Lisboa: Gradiva, 1998.

TULLIS, T.; ALBERT, W. Measuring the user experience: collecting, analyzing, and presenting usability metrics. Burlington: Morgan Kaufman, 2008.

ULRICH, K.; EPPINGER, S. Product Design and Development. $4^{\text {a }}$ ed. New York: Mc 


\section{João Paulo Martins}

João Paulo Martins (Lisboa, 1965)

Licenciado em Arquitetura pela Faculdade de Arquitetura da Universidade Técnica de Lisboa (1988) Mestre em História da Arte pela Faculdade de Ciências Sociais e Humanas da Universidade Nova de Lisboa (1995) Doutor em Arquitetura pela Universidade Técnica de Lisboa (2006)

Assistente da FA-UTL (1996-2006)

Professor Auxiliar da FA-UTL/ FA-ULisboa (2006- )

Tem prática projectual nas áreas do design, interiores e arquitectura, como colaborador do "Atelier Daciano da Costa" (1988-2002) e como associado de "Baixa, Atelier de Arquitetura" (2006-2013).

Comissário da exposição "Daciano da Costa, Designer" (Fundação Calouste Gulbenkian, 2001).

Investigador principal do projecto de investigação "Móveis Modernos. A actividade da Comissão para Aquisição de Mobiliário no âmbito da Direcção-Geral dos Edifícios e Monumentos Nacionais. 1940-1980" com financiamento da FCT (PTDC/AUR-AQI/115660/2009) Comissário da exposição "O respeito e a disciplina que a todos se impõe. Mobiliário para edifícios públicos em Portugal. 1934>1974", patente no MUDE, Museu do Design e da Moda, Coleção Francisco Capelo.

Investigador do projecto de investigação “Design em Portugal (1960-1974): acções, intervenientes e repercussões do Núcleo de Arte e Arquitectura Industrial e do Núcleo de Design Industrial do Instituto Nacional de Investigação Industrial (I.N.I.I.)", com financiamento da FCT (PTDC/EATHAT/121601/2010).

\section{António Marques Pinho}

1981 - Licenciatura em Eng. Mecânica pela Faculdade de Eng. da Universidade do Porto

1985 - Mestrado em Eng. Estrutural pela Faculdade de Eng. da Universidade do Porto

1996 - Doutoramento em Eng. Mecânica, especialidade de Mecânica dos Materiais, pela Universidade do Minho

1981-2013 - Professor Auxiliar do Departamento de Eng. Mecânica da Universidade do Minho, desde 19 de Junho de 2001, com contrato por 
Graw Hill. 2008.

tempo indeterminado.

Sócio das empresas Cert\&Mec e iSurgical3D, sediadas no Avepark.

Como sócio da iSurgical3D é responsável pela área da mecânica nomeadamente no que diz respeito ao desenvolvimento dos sistemas produzidos pela empresa, tais como os dispositivos médicos.

Foi regente e docente de mais 2 dezenas disciplinas das licenciaturas e mestrados em Engenharia Mecânica, Têxtil, Materiais, Produção e Biomédica.

Orientou mais de 30 estágios curriculares da licenciatura em Eng. de Mecânica. Orientou mais de 2 dezenas de dissertação de mestrado, nas áreas de Mecânica e Biomédica. Orientou, também, dois Estágios Formais pela Ordem dos Engenheiros.

Foi responsável pelo grupo disciplinar de Mecânica dos Materiais.

A actividade científica foi desenvolvida enquanto membro do Centro de Eng. Mecânica da UM e do Instituto de Materiais (IMAT-Minho) até final de 2001. Entre 2001 e 2007 passou a integrar o centro CITEPE. Desde 2007 integra o centro CT2M, centro integrado na FCT.

Integra/integrou as equipas de investigação de mais de duas dezenas de projetos financiados por entidades públicas nacionais e internacionais apresentando, como exemplo, apenas:

Aplicação da Mecânica do Impacto no Estudo de Componentes Estruturais em Aços de Alta Resistência, POCTI/EME/39389/2001, coordenador do projecto desde 01-05-2002 a 30/9/2005, (financiamento 50.000 €).

Integra a equipa do projeto NPMimetic - Biomimetic nano-fiber-based nucleus pulposus regeneration for the treatment of degenerative disc disease. Início:01-02-2011, Fim:31-01-2015, Programa: 7th. Framwork Programme. Orçamento Total: $€ 3748$ 000. 2/2

Integra a equipa do projeto HPTB - High Performance Torsion Beam. Início:01-02-2011, Fim:31-01-2015, Programa: QREN. Orçamento Total: € 1 784658.

Foi o orientador científico de cinco doutores, que concluíram os doutoramentos em 2002, 2004, dois em 2008 e outro em 2009:

Atualmente éorientador de 4 doutoramentos em curso, 3 pela Universidade do Minho e 1 pela Universidade Técnica de Lisboa. Um sendo engenheiro do quadro da EFACEC e que tem aprovado um financiamento da FCT, BDE. 


\section{Ana Margarida Ferreira}

Ana Margarida Ferreira (Évora, 1969)

É licenciada em Design, opção Design Industrial (1995, IADE), pós-graduada em Design de Produto (1996, Glasgow School of Art/CPD) e doutorada em Engenharia da Produção/Design (2008, UBI).

Professora Auxiliar no IADE-U, Coordenadora da Licenciatura em Design e Coordenadora do Grupo de Investigação ID:Co.Lab da UNIDCOM/IADE. É ainda investigadora do LOGO/Universidade Federal Santa Catarina.

Coordena e participa em projectos de investigação multidisciplinares que ligam áreas como o Design, o Artesanato, a Engenharia, a Saúde, o Turismo

e o Património e visam a Inovação Sustentável, o Empreendedorismo Social e o desenvolvimento do Território. A sua investigação enquadrase no âmbito das Indústrias Culturais e Criativas. É regente e lecciona(ou) as unidades curriculares de Design e Inovação e Seminários de Grupos de Investigação em Design (Doutoramento), de Metodologia de Investigação, Design e Inovação e Design e Sustentabilidade (Mestrado) e Design de Produção e Criatividade e Inovação (Licenciatura). É membro dos Conselhos Científico e Pedagógico do IADE-U e do Conselho Directivo da UNIDCOM/IADE.

Foi Directora Executiva da UNIDCOM/IADE (unidade de I\&D) e VicePresidente do Conselho de Direcção do IADE. Faz consultoria de projectos de Design e de I\&D na mesma área.

Orienta 5 trabalhos de doutoramento e vários de mestrado.

Atualmente tem como Principais interesses de investigação: Design, Criatividade e Inovações Sustentáveis; Saúde, Turismo, Bem-Estar e Território; Indústrias Culturais e Criativas; Mudança Social.

\section{Demétrio Ferreira Matos}

Demétrio Ferreira Matos (França, 1977)

Licenciado em Design de Equipamento pela Escola Superior de Artes e Design de Matosinhos (2001).

Mestre em Design Industrial pela Faculdade de Engenharia da Universidade do Porto (2009).

A frequentar o doutoramento em Design na Faculdade de Arquitectura da Universidade de Lisboa 
Recebido em: 07/04/2014; Aceito em: 12/04/2014.

Esta obra foi licenciada com uma Licença Creative Commons.
Professor convidado da Escola de Tecnologia do Instituto Politécnico Do Cávado e do Ave. Departamento: Design Docentes, no $1^{\circ} \& 2^{\circ}$ ciclos de estudos, de unidades curriculares de Projeto de Design Industrial, Modelação 3D, Ergonomia e Desenho Assistido por Computador. Investigador do Grupo de Investigação ID:Co.Lab da UNIDCOM/IADE, e do Centro de investigação em Arquitetura, Urbanismo e Design (CIAUD) da Faculdade de Arquitetura da Universidade de Lisboa (FA/Ulisboa). Investigador e orientador científico em projecto de investigação focados na melhoria de qualidade de vida e melhoria de dispositivos médicos. 